\title{
SAÚDE DO HOMEM: IDENTIFICAÇÃO E ANÁLISE DOS FATORES RELACIONADOS À PROCURA, OU NÃO, DOS SERVIÇOS DE ATENÇÃO PRIMÁRIA
}

\author{
Viviane Santos Mendes Carneiro \\ Raphael Neiva Praça Adjuto² \\ Kelly Aparecida Palma Alves ${ }^{3}$
}

CARNEIRO, V. S. M.; ADJUTO, R. N. P.; ALVES, K. A. P. Saúde do homem: identificação e análise dos fatores relacionados à procura, ou não, dos serviços de atenção primária. Arq. Cienc. Saúde UNIPAR, Umuarama, v. 23, n. 1, p, 35-40, jan./abr. 2019.

\begin{abstract}
RESUMO: Identificar e analisar os fatores que dificultam a procura dos serviços de Atenção Primária relatados pela população masculina. Trata-se de um estudo descritivo com abordagem quantitativa. As informações foram coletadas por meio de aplicação de questionário estruturado com perguntas objetivas e subjetivas para 384 homens com idade entre 18 e 59 anos. Identificou-se a participação majoritária $(33,85 \%)$ de indivíduos com idade entre 18 e 24 anos. A visão dos entrevistados acerca de sua saúde e gênero foi de que o homem é mais resistente, forte e invulnerável. Os principais motivos impeditivos relacionados a não procura dos serviços de Atenção Primária foram: preferência por se automedicar, falta de tempo, e desconforto dentro da Unidade Básica de Saúde. A entrada dos homens no sistema de saúde, no ano anterior à pesquisa, ocorreu principalmente pela atenção ambulatorial e hospitalar de média e alta complexidade (62,2\%). Para a adoção da Atenção Primária como porta de entrada dos homens no Sistema Único de saúde, é preciso mudar a visão de que os serviços de saúde têm como único propósito tratar as doenças. Essa sensibilização deve começar mediante conhecimento sobre as características sociais, econômicas e culturais dos homens que receberão a atenção à saúde, além do preparo da equipe de saúde e gestores, seguido pelo acolhimento e busca ativa dessa população.
\end{abstract}

PALAVRAS-CHAVE: Saúde do Homem. Identidade de Gênero. Atenção Primária.

\section{MEN'S HEALTH: IDENTIFICATION AND ANALYSIS OF FACTORS RELATED TO THE DEMAND FOR PRIMARY CARE} SERVICES

\begin{abstract}
This study aims at identifying and analyzing the factors that hinder the demand for Primary Care services reported by the male population. It is a descriptive study with a quantitative approach. The information was collected through the application of a structured questionnaire with objective and subjective questions, applied to 384 men aged between 18 and 59 years. It could be observed that most participants (33.85\%) were aged between 18 and 24 years. The view of the interviewees about their health and gender was that men are more resilient, stronger and invulnerable. The main impediments regarding their not seeking primary care services were: preference for self-medication, lack of time, and discomfort with the Basic Health Unit. The entry of men into the health system in the year prior to the survey occurred mainly by outpatient and hospital care of medium and high complexity (62.2\%). For the adoption of Primary Care Service as the entry point for men in the Unified Health System, it is necessary to change the behavior of the male population in relation to their own health. It is necessary to change the view that health services have the sole purpose of treating diseases. This awareness should begin with knowledge about the social, economic and cultural characteristics of men who will receive health care, as well as the preparation of the health team and managers, followed by the reception and active search of that population.
\end{abstract}

KEY WORDS: Human Health. Gender Identity. Primary attention.

\section{Introdução}

A Política Nacional de Atenção Integral à Saúde do homem, instituída pela portaria $\mathrm{N}^{\mathrm{o}} 1.944$ de 27 de agosto de 2009, está alinhada com a Política Nacional de Atenção Básica e a Equipe Estratégia Saúde da Família, que são as portas de entrada prioritárias do Sistema Único de Saúde. Um dos seus principais objetivos é promover ações de saúde que contribuam significativamente para a compreensão do homem de maneira integral, além de aumentar a expectativa de vida e a redução dos índices de morbimortalidade por causas preveníveis e evitáveis nessa população (BRASIL, 2009b).

Estudo realizado nos Estados Unidos comparou a situação de saúde entre homens e mulheres, e descreveu que os homens têm se mostrado mais vulneráveis às doenças, principalmente aquelas graves e crônicas, e que tem expectativa de vida menor que a das mulheres em cerca de sete anos. Ainda segundo este trabalho, as crenças e comporta- mentos relacionados à saúde são fatores que contribuem para esse resultado (COURTENAY, 2000). Os homens têm maior dificuldade em adotar comportamentos saudáveis e que não apresentem riscos a sua saúde. Apesar dessa maior vulnerabilidade e das altas taxas de mortalidade, os homens não procuram os serviços preventivos, mais comumente procurados pelas mulheres, e adentram o sistema pela atenção hospitalar de média e alta complexidade. Essa atitude tem gerado como consequência o agravo das morbidades, que poderiam ser evitados caso os homens realizassem, com regularidade, as medidas de prevenção primária (FIGUEIREDO, 2005).

As variáveis culturais influenciam em grande parte sobre a não adesão às medidas de atenção integral. Nesse sentido, pode-se afirmar que ao se entender a categoria gênero, haverá maior compreensão sobre as questões relacionadas à saúde masculina (SCOTT, 1995). Gênero pode ser compreendido como uma construção social da diferença sexual, envolvendo homens e mulheres, e constrói-se em cima de

DOI: 10.25110 /arqsaude.v23i1.2019.6521

${ }^{1}$ Enfermeira. Especialista em Urgência e Emergência pelo Programa de Residência Multiprofissional em Saúde do Hospital das Clínicas da Universidade Federal de Goiás e Especialista em Gestão em Enfermagem. E-mail: vsm.mendes@gmail.com

${ }^{2}$ Enfermeiro. Especialista em Terapia Intensiva pelo Programa de Residência Uniprofissional da Escola Superior de Ciência da Saúde e Especialista em Saúde Coletiva. E-mail: raphael.adjuto@gmail.com

${ }^{3}$ Enfermeira. Mestre em Ciências da Saúde pela Universidade de Brasília. E-mail: kelly.escs@gmail.com 
valores que remontam às vivências na vida cotidiana. A partir desses valores, homens e mulheres determinam atitudes que acreditam ser o esperado socialmente do que venha a ser um bom homem ou uma boa mulher, e adotam esse comportamento (FIGUEIREDO; SCHRAIBER, 2011).

Um dos principais desafios da Atenção Primária é a ampliação do acesso da população masculina aos serviços de saúde. O aprofundamento de estudos sobre gênero masculinidade e fatores que dificultam a procura dos serviços pode fornecer ferramentas que possibilitem a construção de um caminho para solucionar esse desafio.

$\mathrm{O}$ objetivo dessa pesquisa foi identificar e analisar os fatores que dificultam e/ou impedem a procura da população masculina pelos serviços de Atenção Primária, levando em consideração sua visão acerca de sua própria saúde e masculinidade, bem como os serviços oferecidos na Atenção Básica.

\section{Material e Método}

Trata-se de uma pesquisa descritiva com abordagem quantitativa, realizado em uma rodoviária localizada em Brasília - DF, durante o período de agosto a outubro de 2014. A população do estudo constituiu-se de homens com faixa etária entre 18 e 59 anos que passaram pela rodoviária e aceitaram participar da pesquisa. A escolha da faixa etária teve como fundamento excluir os adolescentes e os idosos com a intenção de estudar somente a população masculina adulta.

A rodoviária recebe cerca de 700 mil passageiros por dia. Além das linhas de ônibus que circulam dentro dos limites do Distrito Federal. O terminal recebe linhas interurbanas que ligam Brasília aos municípios vizinhos em Goiás. O local foi escolhido devido à quantidade de pessoas que passam por ele, com a intenção de se obter uma amostra diversificada. A amostra é do tipo aleatório simples. Para seu cálculo considerou-se um erro amostral de 5\% e um intervalo de confiança de $95 \%$, totalizando 384 indivíduos.

As informações foram coletadas por meio de aplicação de um questionário estruturado com perguntas objetivas e subjetivas acerca dos seguintes temas: perfil do participante; busca pelos serviços de atenção básica e hospitalar; e dificuldades para buscar os serviços de saúde. Os dados coletados foram inseridos em planilha no programa Microsoft Office Excel ${ }^{\circledR} 2010$. Os resultados se deram a partir da tabulação dos dados no mesmo programa, utilizando frequência simples. A discussão foi feita comparando os resultados encontrados nessa pesquisa com os resultados encontrados na literatura sobre o tema.

A realização deste estudo seguiu os preceitos éticos estabelecidos na Resolução $\mathrm{n}^{\circ} 466$ de 12 de dezembro de 2012 que trata sobre pesquisas e testes realizados em seres humanos. A coleta de dados somente teve início após a aprovação do projeto de pesquisa pelo Comitê de Ética em Pesquisa da Universidade Católica de Brasília sob o número CAAE 5380013.4.0000.0029. Todos os participantes assinaram o termo de consentimento livre e esclarecido.

\section{Resultados}

A população amostral deste estudo foi de 384 homens distribuídos de acordo com a tabela 1 .
Tabela 1: Distribuição dos homens de acordo com idade, estado civil, cor e escolaridade, no período de $1^{\mathrm{a}}$ de a agosto a 31 de outubro de 2014, Distrito Federal, 2018.

\begin{tabular}{lcc}
\hline \multicolumn{1}{c}{ Características } & n (384) & \% \\
\hline Idade & & \\
18 a 24 & 130 & 33,85 \\
25 a 29 & 73 & 19,01 \\
30 a 39 & 83 & 21,61 \\
40 a 49 & 58 & 15,10 \\
50 a 59 & 38 & 9,89 \\
Não responderam & 02 & 0,52 \\
TOTAL & 384 & 100
\end{tabular}

\section{Estado civil}

$\begin{array}{lcc}\text { Solteiro } & 207 & 53,90 \\ \text { Casado } & 169 & 44,01 \\ \text { Divorciado } & 05 & 1,30 \\ \text { Viúvo } & 01 & 0,26 \\ \text { Não responderam } & 02 & 0,52 \\ \text { TOTAL } & 384 & 100\end{array}$

Cor

$\begin{array}{lll}\text { Branco } & 132 & 34,37\end{array}$

$\begin{array}{lll}\text { Pardo } & 175 \quad 45,57\end{array}$

Amarelo $\quad 23 \quad 5,98$

Negro $\quad 52 \quad 13,54$

Não responderam $\quad 02 \quad 0,52$

$\begin{array}{lll}\text { TOTAL } & 384 & 100\end{array}$

Escolaridade

$\begin{array}{lll}\text { Analfabeto } & 01 & 0,26\end{array}$

Analfabeto Funcional $\quad 02 \quad 0,52$

Ensino fundamental incompleto $\quad 14 \quad 3,64$

Ensino fundamental completo $\quad 21 \quad 5,46$

Ensino médio incompleto $\quad 19 \quad 4,94$

Ensino médio completo $\quad 110 \quad 28,64$

$\begin{array}{lll}\text { Ensino superior incompleto } & 89 & 23,17\end{array}$

Ensino superior completo $\quad 126 \quad 32,81$

Não responderam $\quad 02 \quad 0,52$

TOTAL $384 \quad 100$

Fonte: Dados coletados pelos autores

Quando questionados sobre quem eles acreditavam procurar mais os serviços primários de saúde, 363 homens $(94,53 \%)$ informaram que são as mulheres. Os homens que afirmaram ser a mulher quem mais procura os serviços de saúde justificaram da seguinte forma: ela é mais cuidadosa, preocupada e consciente com a própria saúde. Outros afirmaram que a mulher é mais frágil e vulnerável às doenças; a mulher é acostumada desde cedo a procurar os serviços de saúde e fazer prevenção, e que esse ensino faz parte da cultura dela; a mulher tem mais tempo; existem mais políticas públicas voltadas para as mulheres, mais facilidade de acesso e mais especialistas; o homem deixa de procurar por preconceito, machismo, medo, e por achar que é "frescura" procurar 
assistência médica.

A tabela 2 apresenta a relação de unidades de saúde mais próximas das residências dos entrevistados. Mais da metade da população entrevistada informou que a Unidade Básica de Saúde era a mais próxima.

Tabela 2: Relação de unidades de saúde mais próximas das residências dos entrevistados, no período de $1^{\mathrm{a}}$ de a agosto a 31 de outubro de 2014, Distrito Federal, 2018.

\begin{tabular}{lcc}
\hline \multicolumn{1}{c}{ Unidade de saúde mais próxima } & n (384) & $\mathbf{\%}$ \\
\hline Unidade Básica de Saúde (UBS) & 238 & 61,97 \\
Hospital & 138 & 35,93 \\
UBS e Hospital & 07 & 1,82 \\
Não tem & 01 & 0,26 \\
\hline
\end{tabular}

Fonte: Dados coletados pelos autores

Entretanto, apesar da Unidade Básica de Saúde (UBS) ser a unidade de saúde mais próxima, ao serem questionados sobre a procura de assistência de saúde, o hospital foi o local mais procurado, conforme a Tabela 3 .

Tabela 3: Relação de homens que procuraram assistência à saúde no último ano, no período de $1^{\text {a }}$ de a agosto a 31 de outubro de 2014, Distrito Federal, 2018.

\begin{tabular}{|c|c|c|c|c|c|}
\hline \multirow{2}{*}{$\begin{array}{c}\text { Quantas vezes procurou os } \\
\text { serviços de saúde }\end{array}$} & \multirow{2}{*}{$\mathbf{n}$} & \multirow{2}{*}{$\%$} & \multirow{2}{*}{$\begin{array}{c}\text { 2014, Distrito Federal, } 2018 . \\
\text { Pergunta }\end{array}$} & \multirow[b]{2}{*}{$\mathrm{n}$} & \multirow[b]{2}{*}{$\%$} \\
\hline & & & & & \\
\hline$U B S(n=384)$ & & & Possui alguma doença $(n=384)$ & & \\
\hline Nenhuma & 214 & 55,72 & $\operatorname{Sim}$ & 66 & 17,18 \\
\hline Uma vez & 55 & 14,32 & Não & 318 & 82,81 \\
\hline Duas vezes & 51 & 13,28 & TOTAL & 384 & 100 \\
\hline Três vezes & 35 & 9,11 & Como descobriu: $(n=66) U B S$ & 04 & 6,06 \\
\hline Quatro vezes & 07 & 1,82 & Farmácia & 05 & 7,57 \\
\hline Cinco vezes & 07 & 1,82 & Hospital & 48 & 72,72 \\
\hline Mais de cinco vezes & 15 & 3,90 & Outro & 08 & 12,12 \\
\hline TOTAL & 384 & 100 & Não respondeu & 01 & 1,51 \\
\hline Foi atendido? $(n=170)$ & & & TOTAL & 66 & 100 \\
\hline $\operatorname{Sim}$ & 165 & 97,05 & Realiza o acompanhamento $(n=66)$ & & \\
\hline Não & 04 & 2,35 & $\operatorname{Sim}$ & 51 & 77,27 \\
\hline Não respondeu & 01 & 0,58 & Não & 15 & 22,72 \\
\hline TOTAL & 170 & 100 & TOTAL & 66 & 100 \\
\hline Voltaria? $(n=170)$ & & & Com que frequência $(n=51)$ & & \\
\hline Sim & 145 & 85,29 & A cada 2 anos & 02 & 3,92 \\
\hline Não & 23 & 13,52 & Anualmente & 13 & 25,49 \\
\hline Não responderam & 04 & 2,35 & Semestralmente & 15 & 29,41 \\
\hline TOTAL & 170 & 100 & De 4 em 4 meses & 02 & 3,92 \\
\hline Hospital $(n=384)$ & & & Trimestralmente & 06 & 11,76 \\
\hline Nenhuma & 145 & 37,76 & Bimestralmente & 03 & 5,88 \\
\hline Uma vez & 89 & 23,17 & Mensalmente & 09 & 17,64 \\
\hline Duas vezes & 67 & 17,44 & Não respondeu & 01 & 1,96 \\
\hline Três vezes & 37 & 9,63 & TOTAL & 51 & 100 \\
\hline Quatro vezes & 16 & 4,16 & Onde faz o acompanhamento $(n=51)$ & & \\
\hline Cinco vezes & 09 & 2,34 & UBS & 12 & 23,52 \\
\hline
\end{tabular}

Mais de cinco vezes $\quad 21 \quad 5,46$

$\begin{array}{lll}\text { TOTAL } & 384 & 100\end{array}$

Foi atendido? $(n=239)$

Sim

22594,14

Não $13 \quad 5,43$

Não respondeu $\quad 01 \quad 0,41$

TOTAL $239 \quad 100$

Voltaria? $(n=239)$

Sim $202 \quad 84,51$

Não $\quad 36 \quad 15,06$

Não respondeu $\quad 01 \quad 0,41$

$\begin{array}{lll}\text { TOTAL } & 239 & 100\end{array}$

Fonte: Dados coletados pelos autores

O hospital foi o local onde a maior parte dos pacientes portadores de doenças crônicas recebeu o diagnóstico, além de ser a instituição de preferência para o acompanhamento da patologia.

Tabela 4: Relação de quem possuía algum tipo de doença crônica, local da descoberta, forma de acompanhamento e frequência, no período de $1^{\text {a }}$ de a agosto a 31 de outubro de 2014, Distrito Federal, 2018 


\begin{tabular}{lcc} 
Farmácia & 02 & 3,92 \\
Hospital & 32 & 62,74 \\
Outro & 05 & 9,80 \\
TOTAL & 51 & 100 \\
\hline
\end{tabular}

Fonte: Dados coletados pelos autores

Outra questão analisada foram os motivos e/ou fatores que dificultam a procura pelos serviços de Atenção Primária, demonstrado na tabela 5.

Tabela 5: Motivos que interferiram na busca aos serviços de saúde, no período de $1^{\text {a }}$ de a agosto a 31 de outubro de 2014, Distrito Federal, 2018.

\begin{tabular}{lcc}
\hline \multicolumn{1}{c}{ Motivos que interferem: } & n & $\mathbf{\%}$ \\
\hline Não conhece UBS & 26 & 6,77 \\
Prefere automedicar-se & 122 & 31,77 \\
Não se sente à vontade na UBS & 82 & 21,35 \\
Acha que as UBS são espaços mais & 37 & 9,63 \\
femininos & 86 & 22,39 \\
Falta de tempo & 39 & 10,15 \\
Têm medo de descobrir que possui & & 43,22 \\
doença grave & 166 & 14,32 \\
Nenhum motivo interfere & 55 & \\
Outros & &
\end{tabular}

Fonte: Dados coletados pelos autores

\section{Discussão}

Ao se observar os resultados encontrados, é possível identificar a percepção do homem acerca de sua própria saúde e gênero, além de observar respostas que caracterizam o gênero feminino. Para os entrevistados, o homem é um ser invulnerável, forte, tem menos probabilidade de ter doenças, e diz que buscar os serviços de saúde, principalmente para prevenção, é um sinal de fraqueza. A mulher procura mais os serviços de saúde por ser mais preocupada e interessada, frágil, mais susceptível às doenças, e por ter enraizada na cultura a necessidade de acompanhamento médico. Essa ideia é afirmada por outro estudo (GOMES et al., 2011), que descreve o homem de acordo com a visão masculina: forte, cheio de vida, sem direito a se queixar e demonstrar fraqueza, e coloca a mulher como o sexo frágil, que necessita de atenção e cuidado. Ressalta-se a questão da invulnerabilidade e da busca de risco como um valor, e do cuidado à saúde relacionado à mulher (COUTO et al., 2010).

Por se sentirem dessa forma, os homens podem não adotar comportamentos preventivos, tampouco buscar os serviços de saúde. Esse senso de masculinidade dificulta a adoção de hábitos e convicções mais saudáveis e leva o homem a acreditar numa ilusão de que ele não adoece (BERHAME et al., 2002).

Estudo realizado nos Estados Unidos comparou a situação de saúde entre homens e mulheres, e descreveu que os homens têm se mostrado mais vulneráveis às doenças, principalmente aquelas graves e crônicas, e que tem expectativa de vida menor que a das mulheres em cerca de sete anos. Ainda segundo este trabalho, as crenças e comportamentos relacionados à saúde são fatores que contribuem para esse resultado (COURTENAY, 2000).

Os homens têm maior dificuldade em adotar comportamentos saudáveis e que não apresentem riscos a sua saúde. Apesar dessa maior vulnerabilidade e das altas taxas de mortalidade, os homens não procuram os serviços preventivos, mais comumente procurados pelas mulheres, e adentram o sistema pela atenção hospitalar de média e alta complexidade. Essa atitude tem gerado como consequência o agravo das morbidades, que poderiam ser evitados caso os homens realizassem, com regularidade, as medidas de prevenção primária (FIGUEIREDO, 2005).

Nesta pesquisa, grande maioria dos homens entrevistados afirmou ser a mulher quem procura mais os serviços de saúde. Entre as principais justificativas, estão as citadas acima, referentes à cultura e gênero de ambos os sexos. Outra justificativa foi a facilidade de acesso, por conta das inúmeras políticas e programas voltados para elas. A ampliação do acesso aos serviços de saúde pelas mulheres pode estar relacionada ao enfoque ao câncer de mama, de colo de útero e questões ligadas à gravidez. Além de assuntos como tensão pré-menstrual, climatério e/ou menopausa serem temas recorrentes entre as mulheres. Existem poucas campanhas voltadas à população masculina, que parecem não estar preocupadas em sensibilizar e estimular novos hábitos nessa população (GOMES; NASCIMENTO; ARAÚJO, 2007).

Na percepção masculina, os serviços públicos costumam ser um espaço mais feminino. Isto pode ocorrer devido a cartazes de campanhas sobre amamentação, câncer de mama e colo do útero, decorações femininas feitas pelas próprias funcionárias, pela UBS ser frequentada por um número bem maior de mulheres do que de homens e por, geralmente, possuir uma equipe composta, em sua maioria, por mulheres. Essas situações provocariam nos homens uma sensação de não pertencimento àquele espaço, fazendo com que eles não se sintam à vontade e, como consequência, deixem de procurar e usar os serviços ali oferecidos (GOMES; NASCIMENTO; ARAÚJO, 2007).

Além desses, vários outros motivos são citados quando se discute a dificuldade do homem em procurar os serviços de atenção primária. Vários estudos (FIGUEIREDO, 2005; GOMES; NASCIMENTO; ARAÚJO, 2007; COUTO et al., 2010; BRITO; SANTOS; MACIEL, 2010) destacam que uma dessas dificuldades está relacionada à falta de tempo da população masculina em procurar esse tipo de serviço. Em seus estudos, o principal motivo para falta de tempo é o trabalho. Culturalmente, o homem é visto como o provedor da casa e não pode correr o risco de perder um dia de trabalho. Neste estudo, 22,39\% dos homens informaram que a falta de tempo interfere na procura dos serviços de saúde. Entre os motivos colocados estão: trabalho, estudos e demora no atendimento. Essa demora desmotiva a população masculina, que considera uma perda de tempo e, quase sempre, sem real solução dos seus problemas.

Muitas vezes a não busca pelos serviços de atenção primária acontece pela falta de conhecimento sobre esses serviços. A população não conhece a UBS, articulada juntamente com a Estratégia Saúde da Família (ESF), como porta de entrada do SUS (BRASIL, 2009a). Sendo assim, já procuram, primeiramente, o hospital. Essa falta de conhecimento é responsável pela procura dos serviços de média e alta com- 
plexidade, contribuindo, assim, para a permanência da visão curativista das instituições de saúde.

Entendendo a assistência à saúde de forma curativista, os homens preferem dar prioridade aos hospitais e prontos-socorros por acreditarem que o atendimento deve ser rápido e pontual (SCHRAIBER; GOMES; COLTO, 2005). E quando não possuem nenhum problema de saúde aparente ou que incomode, acreditam não haver necessidade de procurar pelos serviços de atenção primária, como relatado pelos entrevistados nesta pesquisa. A prevenção e a promoção da saúde são vistas como algo inerente às mulheres, podendo ser compreendidas como parte da concepção tradicional de gênero, em que o cuidado e a preocupação com a saúde estão ligados ao gênero feminino (SCHRAIBER et al., 2010).

Foi observado nesta pesquisa que a unidade de saúde mais próxima da residência dos participantes é a UBS e, em segundo lugar, os hospitais. Entretanto, apesar da UBS ser mais perto, os homens procuraram mais os hospitais. A não procura pela atenção básica limita o acesso às ações de prevenção primária, quando muitas doenças poderiam ser evitadas.

A maior procura pelos hospitais é evidenciada quando analisados os resultados referentes à existência de alguma doença crônica, local de descoberta e forma de acompanhamento. A maioria dos participantes portadores de doenças crônicas relataram que receberam o diagnóstico dentro do ambiente hospitalar, e que este é o local onde realizam o acompanhamento da doença. Dessa forma, é possível observar a predileção dos homens pelos serviços de média e alta complexidade, sendo que as doenças citadas poderiam ser acompanhados na atenção primária.

O grande desafio, quando se fala em homens na Atenção Primária, é fazer com que eles se tornem adeptos a este tipo de serviço. Compreender como os homens constroem sua masculinidade e entender a questão de gênero é essencial para ajudar a criar mecanismos de acolhimento desse grupo populacional (DUARTE; OLIVEIRA; SOUZA, 2012). Os estereótipos de gênero contribuem para o afastamento da população masculina das UBS. Nesse sentido, tanto a população masculina precisa mudar sua percepção em relação ao cuidado com a própria saúde, quanto aos integrantes da equipe de saúde precisam adotar uma atitude de acolhimento aos homens que procuram os serviços (PONTES et al., 2009).

Sugere-se uma reorganização nas instituições básicas de saúde, no intuito de tornar o serviço mais acolhedor, ágil, resolutivo, gerando assim, uma diminuição nas filas (BRITO; SANTOS; MACIEL, 2010) Além disso, acredita-se que reestruturar as UBS no sentido de estender o seu horário de atendimento para o período noturno oportunizaria a busca por assistência tanto daqueles inseridos no mercado formal de trabalho, como aos trabalhadores autônomos que, muitas vezes, obtêm renda pela quantidade de horas trabalhadas.

As ações da Política Nacional de Atenção Integral à Saúde do Homem buscam promover melhores condições de saúde para a população masculina e objetiva atender esta população na sua integralidade, facilitando e ampliando o acesso desse público aos serviços de saúde (BRASIL, 2009b). Deve-se investir mais na política de saúde do homem, disponibilizando atendimentos específicos à população masculina, educação continuada e maior qualificação dos profissionais, além da realização de campanhas preventivas e promoção da saúde.

\section{Conclusão}

A compreensão de gênero foi identificada como fator para a não adesão desta população a esse tipo de serviço. A opinião de muitos homens é que a doença é considerada como um sinal de fragilidade, característica essa contrária à condição biológica, pois se julgam invulneráveis, fortes e viris, o que acaba contribuindo para que se exponham mais às situações de perigo e acreditem que não há necessidade em procurar serviços preventivos. Motivos como falta de tempo e visão de que a Unidade Básica de Saúde é um espaço mais voltado para as mulheres também devem ser considerados, verificando-se a necessidade de estender o horário de atendimento e realizar campanhas voltadas para a população masculina.

Para a adoção da Atenção Primária como porta de entrada dos homens no Sistema Único de Saúde, é preciso mudar o comportamento da população masculina acerca da própria saúde. É necessário mudar a visão de que os serviços de saúde têm como único propósito tratar as doenças. Essa sensibilização deve começar por meio do conhecimento sobre as características sociais, econômicas e culturais dos homens que receberão a atenção à saúde, além do preparo da equipe de saúde e gestores, seguido pelo acolhimento e busca ativa dessa população.

\section{Referências}

BRASIL. Ministério da Saúde (BR). Departamento de Atenção Básica. Saúde da Família [online]. Brasília, DF, 2009a. Disponível em: <http://dtr2004.saude.gov.br/dab/ atencaobasica.php>. Acesso em: 17 ago. 2017.

BRASIL. Portaria No 1944 de 27 de agosto de 2009 (BR). Institui no âmbito do Sistema Único de Saúde (SUS), a Política Nacional de Atenção Integral à Saúde do Homem. Diário Oficial da União [periódico na internet], 27 ago. 2009b. Disponível em: <http://bvsms.saude.gov.br/bvs/ saudelegis/gm/2009/prt1944 2708 2009.html>. Acesso em: 17 ago. 2017.

BERHAME, Y. et al. Gender, literacy, and survival among Ethiopian adults. Bull World Health Organ, v. 80, n. 9, p. 714-20, 2002.

BRITO, R. S.; SANTOS, D. L.; MACIEL, P. S. Olhar masculino acerca do atendimento na Estratégia Saúde da Família. Rev. Rene, v. 11, n. 4, p. 135-142, 2010.

COURTENAY, W. H. Constructions of masculinity and their influence on men's welleing: a theory of gender and health. SocSciMed, v. 50, n. 10, p. 1385-401, 2000.

COUTO, M. T. et al. O homem na atenção primária à saúde: discutindo (in)visibilidade a partir da perspectiva de gênero. Comunicação Saúde Educação, v. 14, n. 33, p. 257-70, 2010.

DUARTE, S. J. H.; OLIVEIRA, J. R.; SOUZA, R. R. 
A política Saúde do Homem e sua operacionalização na Atenção Primária a Saúde. Revista eletrônica Gestão \& Saúde, v. 3, n. 1, p. 318-17, 2012.

FIGUEIREDO, W. S. Assistência à saúde dos homens: um desafio para os serviços de atenção primária. Ciência \& Saúde Coletiva, v. 10, n. 1, p. 105-09, 2005.

FIGUEIREDO, W. S.; SCHRAIBER, L. B. Concepções de gênero de homens usuários e profissionais de saúde de serviços de atenção primária e os possíveis impactos na saúde da população masculina. Ciência \& Saúde Coletiva, v. 16, n. 1, p. 935-44, 2011.

GOMES, R. et al. Os homens não vêm! Ausência e/ou invisibilidade masculina na atenção primária. Ciência $\&$ Saúde Coletiva, v. 16, n. 1, p. 983-92, 2011.

GOMES, R.; NASCIMENTO, E. F.; ARAÚJO, F. C. Por que os homens buscam menos os serviços de saúde do que as mulheres? As explicações de homens com baixa escolaridade e homens com ensino superior. Cad. Saúde Pública, v. 23, n. 3, p. $565-74,2007$.

PONTES, A. P. M. et al. O princípio de universalidade do acesso aos serviços de saúde: o que pensam os usuários? Escola Ana Nery, v. 13, n. 3, p. 500-07, 2009.

SCHRAIBER, L. B. et al. Necessidades de saúde e masculinidades: atenção primária no cuidado aos homens. Cad. Saúde Pública, v. 26, n. 5, p. 961-70, 2010.

SCHRAIBER, L. B.; GOMES, R.; COUTO, M. T. Homens e saúde na pauta da saúde coletiva. Ciência e Saúde Coletiva, v. 10, n. 1, p. 7-17, 2005.

SCOTT, J. W. Gênero: uma categoria útil da análise histórica. Educação \& Realidade, v. 20, n. 2, p. 71-99, 1995.

Recebido em: 07/12/2017 Aceito em: 29/08/2018 